\title{
Vulvar dystrophies: A long-term Brisbane study of 155 cases
}

\author{
Ian S. C. Jones ${ }^{1 \#}$, Alister Jones ${ }^{2}$ \\ ${ }^{1}$ Women’s and Newborn Services, Royal Brisbane and Women’s Hospital, Brisbane and University of Queensland, Herston, Australia \\ ${ }^{2}$ Department of Surgery, Gold Coast Hospital, Gold Coast, Australia \\ Email: ${ }^{\text {ian_jones@health.qld.gov.au }}$
}

Received 2 May 2012; revised 8 June 2012; accepted 19 June 2012

\begin{abstract}
Objective: To review the long-term outcomes for 155 women with a vulvar dystrophy (VD) who attended the Royal Brisbane Hospital Vulvar Clinic between 1976 and 1988. Methods: VD data from Vulvar Diseases Clinic were reviewed and analysed using the computer software Statistical package for the Social Sciences (SPSS) 11.0. Results: Of 155 patients 94 had Lichen Sclerosus (LS), 41 Lichen Simplex Chronicus (LSC) and 20 Mixed Dystrophy (MD). Three patients developed squamous cell carcinomas of the vulva between 10 and 26 years after presentation with a VD. To date only one of these three patients remains alive following treatment. Conclusion: The need for long term follow up is stressed and any of the three types of VD may become malignant. Time from diagnosis to malignant change is not predictive. VD treatments seem to go through phases with the application of potent steroid creams having stood the test of time.
\end{abstract}

Keywords: Vulvar Dystrophies; Features; Treatment; Malignancy

\section{INTRODUCTION}

Lichen Sclerosus (LS) and Lichen Simplex Chronicus (LSC) are two of the three most common non-neoplastic epithelial disorders of the vulva (the third being lichen planus) [1]. The International Society for the Study of Vulvar Disease (ISSVD) proposed a new nomenclature in 1976. This nomenclature was current when the study cohort was managed and so is used in this article. Since then there have been refinements to this nomenclature [2] with LSC now termed Squamous Cell Hypertrophy and MD termed Lichen Sclerosus with associated Squamous Cell Hypertrophy. However, the histological criteria for diagnosis are unchanged.

\footnotetext{
${ }^{*}$ Conflict of interest: There are no conflicts of interest.

${ }^{\#}$ Corresponding author.
}

The macroscopic features of LS are white flat papules which are scattered or confluent in plaques with atrophy and pallor located on the labia minora, perineum and clitoris. The histological features of LS include a thin, flat epidermis and keratin layer, plus hyalinised dermis bordered inferiorly by a band of chronic inflammatory cells. Elastin fibres are reduced in areas of sclerosis. LSC on the other hand appears as a generalised thickening of the skin of the labia majora. Histologically the epidermis and keratin layer are thickened and the rete pegs increased in number and lengthened. The papillary dermis is thickened and contains a variable chronic inflammatory infiltrate. Mixed dystrophy (MD) combines features of LS and LSC, both macroscopically and histologically [3]. This paper reviews the features, treatment, and incidence of malignant change for 155 women who presented with a vulvar dystrophy to the Vulvar Disease Clinic at the Royal Brisbane Hospital between 1976 and 1988.

\section{MATERIALS AND METHODS}

One hundred and fifty five patients presenting to the Vulvar Diseases Clinic between 1976 and 1988 were diagnosed with VD. They were assessed and then seen annually at either public or private gynaecology clinics. Some patients chose to return to their referring private gynaecologists, hence the need to check on follow up in these cases. During 2010 the medical records from both public and private gynaecology clinics were cross checked with the state wide Queensland Centre for Gynaecological Cancer (QCGC) data base to determine if any VD patient had developed a vulvar malignancy.

Data were stored and analysed using the computer software Statistical Package for the Social Sciences (SPSS) 11.0. Ethics approval for the study was obtained from the Clinical Research Ethics Committee of the Royal Brisbane and Women’s Hospital.

\section{RESULTS}

Of 155 patients 94 had Lichen Sclerosus (LS), 41 Lichen 
Simplex Chronicus (LSC) and 20 Mixed Dystrophy (MD). All age groups were represented in the cohort with the majority being aged 50 years or more. The age range and mean age for the three conditions were for LS 11 88 years (mean 59), LSC 20 - 93 years (mean 59) and MD 56 - 83 years (mean 65). Pruritus was the presenting symptom in $80 \%$ or more for each type of VD. The most common anatomical location for LS was the labia minora, for LSC the labia majora and for MD both sites. Using $\mathrm{z}$ scores these findings were significant $(\mathrm{p}<0.01)$. However it was not possible to equate diagnosis with anatomical location in all cases.

During routine follow up three patients developed squamous cell carcinomas of the vulva between 10 and 26 years after presentation with a VD (Table 1). Of these three patients one came from each of the three types of VD. To date only one patient remains alive and she is without evidence of recurrence of either her vulvar or endometrial cancer. All three patients with vulvar cancer underwent simple vulvectomy and groin node sampling.

In the early part of the study local steroid cream combined with testosterone cream was the most frequently used treatment for LS and steroid cream alone for LSC. Later potent steroid creams became the sole treatment regime for all VDs. Treatment was considered to be successful if the patient was symptom free and the skin appearance returned to normal. However in $20 \%$ of LS cases skin appearance improved but did not return to normal despite regular use of treatment.

\section{DISCUSSION}

The prevalence of female LS is unknown, with estimates ranging from 1:30 to 1:59 women in general gynaecological practice. The highest incidence of LS in women was found post menopause. The differential diagnosis of LS includes lichen planus, with which it may overlap, and vitiligo. The most important risk for these patients is the possibility (between $2 \%-5 \%$ ) of progressing to squamous cell carcinoma [4]. Squamous cell carcinoma of the vulva in younger women is frequently associated with Human Papilloma Virus (HPV) infection and Vulvar Intraepithelial Neoplasia (VIN). This is not usually the case in the older woman who has VIN but no HPV infection [5].

The aetiology of LS is unknown but causation theories abound starting with achlorhydria, the transplant study which suggested local vulval factors facilitated disease expression, the association with auto-immune disease like autoimmune thyroid disorders, vitiligo and alopecia areata, HLA 30/31 and HLA-B4 association, 5 alpha reductase deficiency, acid fast bacterial infection and increased collagen inhibitor enzyme. More recently there is evidence that immunological changes are present in all layers of skin affected by lichen sclerosus. In addition the increased fragility and scarring in skin affected by LS has been explained by the reorganisation of the extracellular matrix due to changes in tenascin, fibrinogen (decrease) and fibronectin (increased) which are important components associated with wound repair.

Lichen simplex chronicus (LSC), describes a nonneoplastic morphological alteration of skin related to chronic irritation with a characteristic histological appearance. LSC is nowadays considered to be a localised form of neurodermatitis with underlying psychological disturbance being present in most cases. The prevalence of LSC is unknown but is estimated to be 1:75 to 1:100 women presenting to a general gynaecological practice (Jones, unpublished work). LSC is a diagnosis of exclusion from other conditions that can cause hyperplastic epithelial changes (e.g. psoriasis, lichen planus, eczema, seborrheic dermatosis, HPV infection and candidiasis).

There are other vulval conditions that can present with

Table 1. Cases that subsequently developed vulvar squamous cell carcinoma.

\begin{tabular}{|c|c|c|c|}
\hline Condition & $\begin{array}{l}\text { Lichen sclerosus } \\
\quad \mathrm{n}=1\end{array}$ & $\begin{array}{l}\text { Lichen simplex chronicus } \\
\qquad \mathrm{n}=1\end{array}$ & $\begin{array}{c}\text { Mixed dystrophy } \\
\mathrm{n}=1\end{array}$ \\
\hline Age when ca found & 1994 aged 78 & 1995 aged 64 & 1994 aged 55 \\
\hline Duration of VD & $26+$ years & $10+$ years & 20 years \\
\hline Location & Labia minora & Clitoris & Labia minora \\
\hline Parity & 0 & 8 & 3 \\
\hline Menopause & aged 35 & Aged 50 & Aged 48 \\
\hline Preceding VIN/atypia & No & Yes, 6 years & Yes, 4 years \\
\hline Current status & $\begin{array}{c}\text { Alive } \\
\text { Dementia } \\
\text { No endometrial ca } \\
\text { No vulva ca }\end{array}$ & Dead of disease & Dead of disease \\
\hline
\end{tabular}


pruritus vulvae for example candidiasis, lichen planus and HPV infection. Most VDs occur in post menopausal women but can occur in the child and during reproductive years. Even pregnant women with their high levels of female hormones can present with VDs suggesting that theories to explain the basis of these conditions can not be solely due to a lack of female hormones.

The study found an association between autoimmune thyroid disease and LS of $10 \%$, which is in line with other reports. There are reports of LS having a hereditary basis with mothers and sisters of sufferers being reported however this was not recognised in the current study. The associations between VDs and diabetes, other skin disorders (psoriasis, vitiligo and lichen planus), drug allergies and non genital cancers did not reach statistical significance. Depression requiring treatment was present in $14 \%$ of LS patients, $29 \%$ of LSC and $20 \%$ of MD. No significant difference was found between these three groups using $\mathrm{z}$ scores (LS v LSC $\mathrm{z}=1.822$; $\mathrm{LS} \mathrm{v} \mathrm{MD} \mathrm{z}=$ 0.338 ; LSC $v$ MD z $=0.44$ ). The benefits of anti depressants include their ability to modify pain.

Of available treatments, most were based on the use of potent glucocorticoid steroid creams, although once symptoms settled the weekly or bi-weekly use of $1 \%$ hydrocortisone proved effective. The concern with long-term use of steroid creams is the atrophying effect this has on vulval skin, especially that affected by LS. Another problem with the use of steroid creams is the development of fungal infection, which is frequently worse in the presence of obesity and diabetes. Steroid creams can also be used in conjunction with vaginal oestrogen cream when vaginal atrophy causes coital difficulties. Steroid cream was a popular and effective treatment for LSC, findings which are in agreement with a series of 976 patients reported by Ayhan et al. [6].

Malignant change in VD patients occurs in between $3 \%-5 \%$. In the current study vulval malignancy was found in three patients. None of the three types of VD were spared from developing malignancy; hence all forms of VD require long term follow up. All three cases of malignancy were post menopausal and those with LS had had their condition for over 20 years (Table 1). Time since menopause for these three patients were 43 years for LS, 14 years for LSC and 7 years for MD, however the duration of VD was not useful in differentiating malignancy risk but the presence of vulval intra epithelial neoplasia (VIN) or cellular atypia was, supporting the need for further biopsies if the skin appearance is suspicious.

\section{ACKNOWLEDGEMENTS}

We thank the Queensland Centre for Gynaecological Cancer (QCGC) staff for their assistance in providing data and checking their data on our study patients.

\section{REFERENCES}

[1] O’Connell, T.X., Nathan, L.S., Satmary, W.A. and Goldstein, A.T. (2008) Non-neoplastic epithelial disorders of the vulva. American Family Physician, 77, 321-326.

[2] Maclean, A.B. (1991) Vulval dystrophy-The passing of a term. Current Obstetrics \& Gynaecology, 1, 97-102. doi:10.1016/0957-5847(91)90038-Y

[3] Neill, S. and Lewis, F. (2009) Ridley's the vulva. 3rd Edition, Wiley-Blackwell, Hoboken.

[4] Smith, Y.R. and Haefner, H.K. (2004) Vulvar lichen sclerosus: Pathophysiology and treatment. American Journal of Clinical Dermatology, 5, 105-125. doi:10.2165/00128071-200405020-00005

[5] Scurry, J.P. and Vanin, K. (1997) Vulvar squamous cell carcinoma and lichen sclerosus. The Australasian Journal of Dermatology, 38, S20-S25.

[6] Ayhan, A., Guvendag Guven, E.S., Guven, S., Sakinci, M. and Kucukali, T. (2006) Medical treatment of vulvar squamous cell hyperplasia. International Journal of Gynaecology \& Obstetrics, 95, 278-283. doi:10.1016/j.ijgo.2006.06.024 